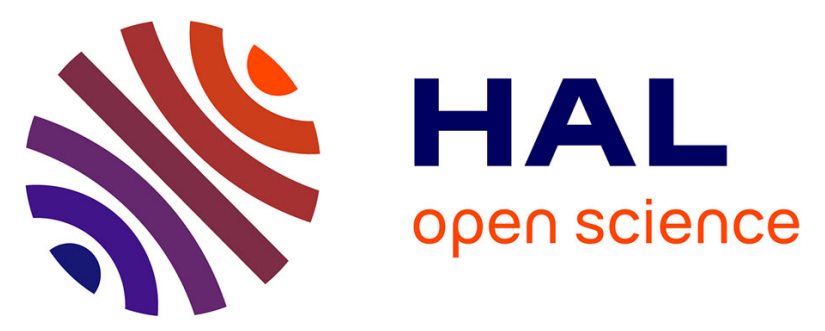

\title{
Proposition of a modal filtering method to enhance heat source computation within heterogeneous thermomechanical problems
}

Thomas Pottier, Hervé Louche, Serge Samper, Hugues Favreliěre, Franck Toussaint, Pierre Vacher

\section{To cite this version:}

Thomas Pottier, Hervé Louche, Serge Samper, Hugues Favreliěre, Franck Toussaint, et al.. Proposition of a modal filtering method to enhance heat source computation within heterogeneous thermomechanical problems. International Journal of Engineering Science, 2014, 81, pp.163 - 176. 10.1016/j.ijengsci.2014.04.010 . hal-00995121

\section{HAL Id: hal-00995121 \\ https://hal.science/hal-00995121}

Submitted on 22 May 2014

HAL is a multi-disciplinary open access archive for the deposit and dissemination of scientific research documents, whether they are published or not. The documents may come from teaching and research institutions in France or abroad, or from public or private research centers.
L'archive ouverte pluridisciplinaire HAL, est destinée au dépôt et à la diffusion de documents scientifiques de niveau recherche, publiés ou non, émanant des établissements d'enseignement et de recherche français ou étrangers, des laboratoires publics ou privés. 


\title{
Proposition of a modal filtering method to enhance heat source computation within heterogeneous thermomechanical problems
}

\begin{abstract}
This paper presents a new approach to evaluate heat sources from thermal field measurements. A modal projection based on dynamics (Discrete Modal Decomposition) is used to estimate the spatial term of a heat diffusion problem. A numerical example is presented in order to validate this approach and compare it to a more classical spectral decomposition (based on thermal considerations). Results show that the proposed projection basis not only provides closer assessment of the heat sources but is also more stable to noise and side effects. Finally, a basis enrichement method is presented and tested. It shows that a priori knowledge of the heat sources shape though approximated (e.g. from strain measurement) enhances the assessement of calorific effects accompanying material deformation.
\end{abstract}

Keywords: Thermal field, Infrared thermography, Spectral projection, Modal decomposition, Heat sources estimation

\section{Introduction}

The study of calorific effects associated with material deformation has been widely investigated since the early works of Lord Kelvin. In the late 90s, experimenters have turned to the use of infrared (IR) thermography in order to estimate the temperature variations at the sample surface during mechanical loading. The observed temperature variations depend on both the material and the type of loading and can, under some assumptions, provide valuable data on the material behaviour. For example, positive temperature variations in one area of a loaded sample may reveal that irreversibilities take place at this location. However, the intensity of the temperature variations does not only depend on the internal mechanisms of the material (reversible or not). They also depends on various characteristics of the thermal problem 
(geometry of the studied sample, thermophysical properties, boundary and initial conditions). Within this context, another approach, based on heat sources analysis, was proposed and has proved its worth on various thermomechanical problems $[1,2,3,4]$.

Hence, heat sources has become relevant quantities to study material behaviour. They provide complementary informations on the instantaneous energy, locally associated with the material behaviour. Either the sign and the magnitude of this volumic power (denoted $w_{c h}^{\prime}$ ) can be linked to the material underlying physics and can also be used to achieve energy balance $[5,6]$.

Classically, the heat sources computation relies on performing both spatial and time derivation of the measured temperature fields $T(x, y, t)$. Under some classical assumptions [2], the 2D-heat diffusion equation is given by

$$
\rho C \frac{\partial \theta}{\partial t}-k\left(\frac{\partial^{2} \theta}{\partial x^{2}}+\frac{\partial^{2} \theta}{\partial y^{2}}\right)=w_{c h}^{\prime} \quad \text { with } \quad \theta(x, y, t)=T-T_{0}
$$

where $\theta$ stands for the temperature variation, $x$ and $y$ are the spatial coordinates, $\rho$ is the mass density, $C$ the thermal capacity, $k$ the thermal conductivity, $T_{0}(x, y)$ the initial temperature field and $w_{c h}^{\prime}$ the internal heat sources. Such a definition allows the assessment of $w_{c h}^{\prime}$ from the time-derivative and the second space-derivative of $\theta(x, y, t)$ and the a priori knowledge of the thermophysical constants $\rho, C$ and $k$.

In practice, the time resolution of modern IR cameras (up to $380 \mathrm{~Hz}$ ) often allows the computation of a proper time derivative of $\theta$ using low-pass or polynomial filters at every pixel. On the other hand, the space-derivative term cannot be estimated as straight-forwardly. The measurement noise, intrinsic to IR-thermography, is amplified by space-derivation operators and massively impairs the calculation of the Laplace terms in Eq. (1).

Many numerical approaches have been developped in the last decade in order to estimate as accurately as possible the left hand side term of Eq. (1). These regularisation methods can be classified into 3 main categories:

- Filtering methods, aimed at supressing the noise from the measurement using Gaussian [7, 8] or low pass filters based on linear, Fourier or wavelet definitions $[2,9,10]$. 
- Inverse methods, aimed at obtaining a quasi-solution through the use of one or several optimization algorithm [11, 12].

- Projection methods, they are among classical means in inverse heat transfer analysis. They consist of decomposing the measurement within a spectral basis built from eigen function of the Laplace operator [2, 13]. These techniques have recently experienced various improvements through the use of wavelet decompositions [14] or branch modes decomposition [15]. This latter approach allows the use of generalized boundary conditions which usually represents the main disadvantage of projection methods. Mixed temporal (Fourier) and spatial (Laplace) projection methods have also been used and provided promising results [16]. Nevertheless, boundary conditions and heat sources reconstruction on the domain contour remains a significant drawback of such approaches.

The present paper proposes to implement a projection method and compare the results obtained using a decomposition built from structural dynamics and another from thermomechanical considerations. The use of a basis, not related to the solution of a given problem, is nowadays among classical means in noise removal approaches $[17,18,19]$. For the sake of comparison, a numerical example is built (section 3 ). The heat sources are then rebuilt using (i) the proposed modal basis (section 2.2) and (ii) the classical spectral basis (section 2.3). Hence, the influence of various parameters along with the measurement noise are investigated (section 4). Finally, a basis enrichement approach is proposed and tested (section 5).

\section{Setup of projection bases}

\subsection{Projection operator}

The thermal field measurement, such as provided by an IR-camera, is a film made of 2-dimensional arrays. The finite resolution of the capture leads to define an integer grid $\mathcal{M}=\mathbb{R}_{\delta} \times \mathbb{R}_{\delta} \cap[0 ; a] \times[0 ; b]$ which step is denoted $\delta$. In addition, the constant frame rate results in the measured field being part of a monotonous discrete sequence:

$$
\theta(x, y)=\left(\theta_{1}(x, y) ; \theta_{2}(x, y) ; \ldots ; \theta_{k}(x, y)\right)
$$


where each time step (denoted using subscript $k \in \mathbb{N}^{*}$ ) is a temperature variation frame.

In a space continuous framework, the use of the modal/spectral approach requires the projection of the measured fields $\theta_{k}(x, y) \in \mathbb{R}$ into an eigen basis $\mathfrak{B} \subset \mathcal{C}^{2}([0 ; a] \times[0 ; b], \mathbb{R})$ independant of the steptime $k$. This basis is defined by its continuous eigen vectors denoted $\left(Q_{i}^{c}\right)_{i \in \mathbb{N}^{*}}$. Hence, assuming that $\mathfrak{B}$ is a basis of the solution subspace $\mathcal{S}$ of Eq. (1), the measured field can be expressed as a linear combination of the eigen vectors:

$$
\theta_{k}^{c}(x, y)=\sum_{i=1}^{+\infty} \alpha_{k, i}^{c} \cdot Q_{i}^{c}(x, y), \quad \forall(x, y) \in \mathbb{R}^{2}
$$

However, assuming a truncation $N$ of the sum in Eq. (3), a residual terms $R_{N}(x, y)$ arises in the second member. This latter tends toward zero on $\mathcal{M}$ if the grid step $\delta$ tends toward zero. In other words it is easy to settle that:

$$
\text { lemma } 1-R_{N}(x, y, \delta) \longrightarrow 0, \quad \forall(x, y) \in \mathbb{R}^{2}
$$

i) if $\delta \longrightarrow 0$

ii) and if $N=|\mathcal{M}|$

where $|\bullet|$ stands for the cardinality of $\bullet$. This results prooves that the space continuous and discrete cases merges when the truncation $N$ equals the number of nodes in $\mathcal{M}$. Hence, if $\mathfrak{B}$ is a basis of the solution subspace $\mathcal{S}$ if Eq. (1) then the projection of $\theta_{k}^{c}$ on $\mathcal{M}$ is exact.

Moreover, one may notice that for a given discretisation $\delta$, the independance of the discrete modal vectors $Q_{i}$ is sufficient to ensure that

lemma $2-R_{N, \delta}(x, y)=0, \quad \forall(x, y) \in \mathcal{M}$

i) if $\mathfrak{B}$ is a basis of the set of function of $\mathcal{M}$ in $\mathbb{R}$,

ii) and if $N=|\mathcal{M}|$.

A consequence of lemma 2 is that if $\mathfrak{B}$ is a free familly (i.e. if $N<$ $|\mathcal{M}|$ ), the residual term is a continuously decreasing function of $N$. Another consequence is that $\mathfrak{B}$ doesn't have to be a basis of $\mathcal{S}$, indeed any independent set of vectors verifies lemma 2. Subsequently, for a given space discretization, if the number of modes equals the number of nodes then the residual can be zero regardless of whether $\mathfrak{B}$ is or isn't a basis of $\mathcal{S}$. This consideration leads to assume that the relation beween the projection basis and the underlying physics is not relevant if $N$ is high enough. 


\subsection{Modal basis related to dynamics}

Let $U$ be a compact subspace of $\mathbb{R}^{3}$ and $q: U \rightarrow \mathbb{R}$ a $\mathcal{C}^{2}$-continuous function. A rectangular domain $U=[0 ; a] \times[0 ; b] \times\left[t_{k} ; t_{k+1}\right]$ designates the solid structure under investigation. The associated geometry (a square plate in the proposed problem) leads to the definition of the following dynamic structural problem:

$$
M \cdot \ddot{q}+K \cdot q=0 \quad \text { with } \quad q=q(x, y, t),
$$

where $M$ and $K$ stand for the mass and the stiffness matrices respectively. Under such formalism, $q(x, y, t)$ stands for the displacements which caracterizes the modal shapes. Such a problem classically gives a frequency based solution

$$
q(x, y, t)=\sum_{i=1}^{+\infty} Q_{i}(x, y) \cdot \cos \left(\omega_{i} t\right),
$$

where $Q_{i}$ is the magnitude vector associated with the pulsation $\omega_{i}$. Hence, in the finite dimension framework, the eigen modes defined by $\left(Q_{i}, \omega_{i}\right)$ are determined by solving the following linear system

$$
\left(M^{-1} K-\frac{1}{\omega_{i}^{2}} \mathbb{I}\right) Q_{i}=0
$$

where $\mathbb{I}$ is the identity matrix and $M^{-1} K$ is of course assumed to be diagonalizable. The discrete solution is computed by using Finite Element Analysis (FEA) and provides the dynamic modal basis $\mathfrak{B}_{D}=\left(Q_{1}, Q_{2}, \ldots, Q_{N}\right)$

The numerical finite element problem is solved using Abaqus software. The procedure can be summarized as following:

1. A square plate is modelled as a 3D deformable shell.

2. The structure is meshed using $100 \times 100$ square quadratic shell elements (9 nodes, normal $z$ ).

3. Boundary conditions are prescribed and impose no in-plane displacement at every nodes, the scalar product of the displacement vector $q(x, y)$ with axis $x$ and $y$ being then: $\langle q(x, y, t) ; x\rangle=0$ and $\langle q(x, y, t) ; y\rangle=$ 
0 everywhere.

4. The frequency solver is run with a requested number of mode of $N=$ 500 (CPU time is 1266s).

5. Modal displacements along $z$ are exported to matlab and interpolated over a $300 \times 300$ monotonic grid (The 25 first modal vectors are ploted in Fig.1-(a)).

However, the non orthonormality of $\mathfrak{B}_{D}$ does not allow the use of the classical projector $P_{Q}=Q Q^{T}$. Indeed, the use of the dual basis $Q^{*}=$ $\left(Q^{T} Q\right)^{-1} Q^{T}$ is required. An infinite norm is given to the modal vectors such as $\left\|Q_{i}\right\|_{\infty}=1$. Thus, the set of modal coordinates $\alpha_{k}$ resulting from the projection of the displacement field at step $k$ (denoted $q_{k}(x, y)$ ) within a non-orthonormal basis is given as follows:

$$
\alpha_{k}=\left\{\alpha_{k, i} \in \mathbb{R}\right\}=\left(Q^{T} Q\right)^{-1} Q^{T} \cdot q_{k}
$$

where $Q$ denotes the matrix whose columns are $Q_{1}, \ldots, Q_{N}$, and $\alpha_{k}=$ $\left(\alpha_{k, i}\right)_{i \in[1 ; N]}$ is the set of modal coordinates. The point of the present paper is to associate the displacement fields $q\left(x, y, t_{k}\right)$ to the measured temperatures $\theta_{k}(x, y)$. Indeed, a consequence of Eq. (6) is that if $N=|\mathcal{M}|$, then $\mathfrak{B}_{D}$ is completed and therefore defines a basis in the solution subspace of Eq. (1) on $\mathcal{M}$. Hence, even though $\mathfrak{B}_{D}$ is far from describing the actual underlaying physics, it may (in theory) lead to an exact solution at nodes if $N=|\mathcal{M}|$. In practice, this amount of modes (e.g. 10000 for a $100 \times 100$ mesh) is of course not relevant from a numerical standpoint, however the obtained basis exhibits several significant advantages. First of all, it naturally describes a space vector and thus allows a straight forward projection. In addition, the energy sorting of modes leads to an increasing complexity of shape (and frequency) when the truncation order $N$ increases. Finally, it does not require the use of variable separation and therefore leads to a slower frequency increasing along $N$. In other words, the $k^{\text {th }}$ mode of $\mathfrak{B}_{D}$ exhibits a smaller frequency than its counterpart in the spectral basis (calculated assuming variable separation). This latter point is of the utmost interest since the computation of the second order space-derivative (finite differences) is well known for significant divergence when high frequencies are involved. 


\subsection{Spectral basis related to the Laplace operator}

Let us consider the two dimensional thermal equation in the rectangle $U=$ $[0 ; a] \times[0 ; b] \times\left[t_{k} ; t_{k+1}\right]$ defined above, with boundary conditions prescribed on the four edges of the rectangle and no heat source. The thermal problem is solved, using Neumann conditions over $\partial U$, as:

$$
\begin{cases}\Delta \theta=\frac{\partial^{2} \theta}{\partial x^{2}}+\frac{\partial^{2} \theta}{\partial y^{2}}=\frac{\rho C}{k} \frac{\partial \theta}{\partial t} & \text { over } U \\ \frac{\partial \theta}{\partial x}(0 ; y, t)=\frac{\partial \theta}{\partial x}(a ; y, t)=0 & \text { over } \partial U_{x} \\ \frac{\partial \theta}{\partial y}(x ; 0, t)=\frac{\partial \theta}{\partial y}(x ; b, t)=0 & \text { over } \partial U_{y}\end{cases}
$$

where $\partial U_{x} \cup \partial U_{y}=\partial U$. Hence, if $\theta$ is solution of this problem, it is an harmonic function. Solutions are found assuming separation of variables. Thus, $\theta(x, y, t)=f(t) u(x) v(y)$. Substituting this in Eq. (8) and dividing by $\theta$ gives:

$$
\frac{\rho C}{k} \frac{\dot{f}}{f}=\left(\frac{u^{\prime \prime}}{u}+\frac{v^{\prime \prime}}{v}\right)
$$

However, the independence of $u, v$ and $t$ to their respective variables imposes those three ratios to be constant. Then, variable separation states the problem as three independant differential equations:

$$
u^{\prime \prime}=\lambda_{1} u \quad \text { and } \quad v^{\prime \prime}=\lambda_{2} v \quad \text { and } \quad \dot{f}=\gamma f,
$$

such as $\lambda_{1}+\lambda_{2}=\gamma$. The shape of the solutions, for $u$ and $v$, depends on the sign of the $\lambda_{i}$ which requires the examination of the two following cases:

$$
\lambda_{i} \leq 0 \Rightarrow\left\{\begin{array}{l}
u(x)=A \cdot \cos \left(\sqrt{\left|\lambda_{1}\right|} x\right)+B \cdot \sin \left(\sqrt{\left|\lambda_{1}\right|} x\right) \\
v(y)=C \cdot \cos \left(\sqrt{\left|\lambda_{2}\right|} y\right)+D \cdot \sin \left(\sqrt{\left|\lambda_{2}\right|} y\right)
\end{array}\right.
$$

or

$$
\lambda_{i}>0 \Rightarrow\left\{\begin{array}{l}
u(x)=\tilde{A} \cdot \cosh \left(\sqrt{\left|\lambda_{1}\right|} x\right)+\tilde{B} \cdot \sinh \left(\sqrt{\left|\lambda_{1}\right|} x\right) \\
v(y)=\tilde{C} \cdot \cosh \left(\sqrt{\left|\lambda_{2}\right|} y\right)+\tilde{D} \cdot \sinh \left(\sqrt{\left|\lambda_{2}\right|} y\right)
\end{array}\right.
$$

Therefore, the boundary conditions of Eq. (8) can be used to determine 
the value of the 8 constants $A_{\lambda}, B_{\lambda}, \ldots, \tilde{D}_{\lambda}$. It comes, that the solutions postulated in Eq. (12) leads to $u(x)=v(y)=0 \forall(x ; y) \in \mathbb{R}^{2}$. Thus, the only solutions postulated in Eq. (11) can be solution of the boundary problem. In such case, it is easy to show that the boundary conditions impose:

$$
\left\{\begin{array}{l}
B=D=0 \\
\lambda_{1}=-\left(\frac{n \pi}{a}\right)^{2} \quad \text { and } \quad \lambda_{2}=-\left(\frac{m \pi}{b}\right)^{2} \\
\gamma=-\left(\frac{n^{2} \pi^{2}}{a^{2}}+\frac{m^{2} \pi^{2}}{b^{2}}\right),
\end{array}\right.
$$

with $n$ and $m$ in $\mathbb{Z}$. According to this expression of $\gamma$, the temporal solution is given by:

$$
f(t)=e^{-\sigma_{n, m} t} \quad \text { with } \quad \sigma_{n, m}=\frac{k \pi^{2}}{\rho C}\left(\frac{n^{2}}{a^{2}}+\frac{m^{2}}{b^{2}}\right) .
$$

Then, assuming a steady state case within $\left[t_{k} ; t_{k+1}\right]$, it can be shown that $f(t)$ can be approximated by 1 . Hence

$$
\theta_{k}(x, y)=\sum_{n=1}^{N} \sum_{m=1}^{N} A_{n} B_{m} \underbrace{\cos \left(\frac{n \pi x}{a}\right) \cos \left(\frac{m \pi y}{b}\right)}_{\text {mode vectors } Q_{n, m}} .
$$

Finally, the vectors of the spectral basis $\mathfrak{B}_{\Delta}=\left(Q_{1,1}, Q_{2,1}, \ldots, Q_{n, m}\right)$ associated with the Laplace operator correspond to the eigenfunctions estimated from the right hand side of Eq. (15). Since the vectors are normalized, $\mathfrak{B}_{\Delta}$ is classically a completed and orthonormal basis on $\mathcal{M}$ [20]:

$$
\left\|Q_{1,1}\right\|_{\infty}=\left\|Q_{2,1}\right\|_{\infty}=\ldots=\left\|Q_{n, m}\right\|_{\infty}=1
$$

In order to sort the obtained basis in increasing order of frequencies, the modes are sorted by increasing products $n \times m$. Double occurences are treated by placing the greater value of $n$ ahead. One of the main consequence of the sorting operation is that it must be perfomed again if $N$ (denoting the total number of modes or truncation order) varies. This disadvantage prevents from a single, once and for all calculation of $\mathfrak{B}_{\Delta}$. Another disadvantage comes from the periodicity of the $Q_{i}$ vectors which requires high truncation order to discribe a non-periodic shape. However, this basis exhibits a major 


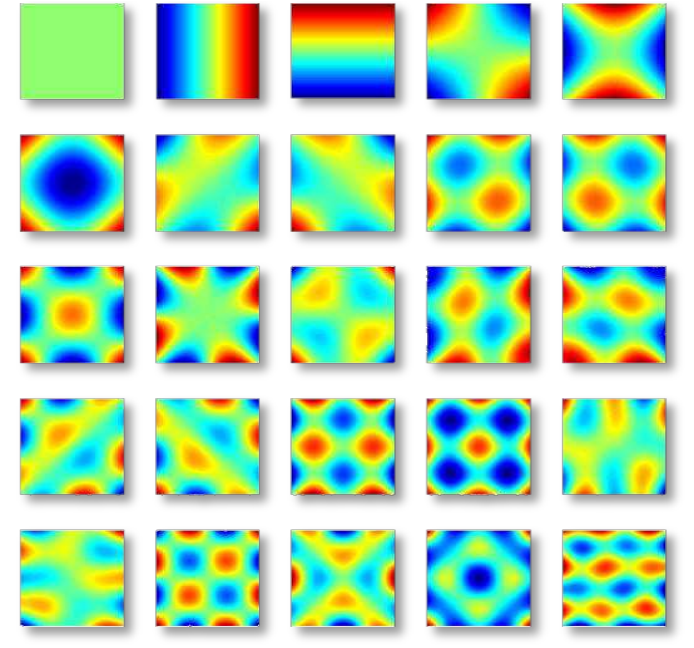

(a)

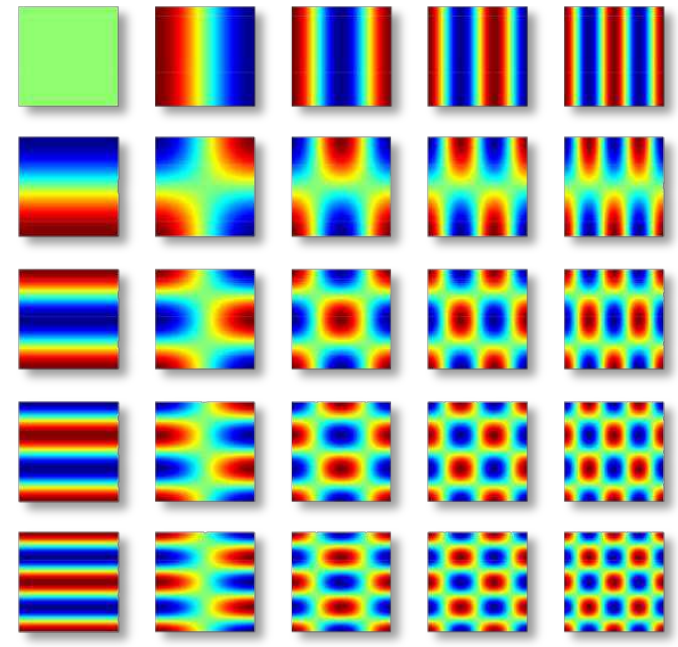

(b)

Figure 1: Shape comparison between modal (a) and spectral (b) projection bases. Only the 25 first modes are presented.

advantage: it is a parabolic solution of Eq. (1) and is therefore close-tophysics.

\subsection{Modes derivation}

As shown in Eq. (1), the measured field must be derived twice in space in order to calculate the Laplace operator. However, since the projector $P_{Q}$ is a linear transformation, the additivity and homogeneity properties allows the writing of the Laplace operator as:

$$
\Delta \theta_{k}(x, y)=\sum_{i=1}^{N} \alpha_{k, i}\left(\frac{\partial^{2} Q_{i}(x, y)}{\partial x^{2}}+\frac{\partial^{2} Q_{i}(x, y)}{\partial y^{2}}\right)+\Delta R_{n, k}(x, y) .
$$

The point is then to assess the second derivative of the mode vectors $Q_{i}$ whether they are stemmed from the modal or the spectral basis. In practice, these basis are evaluated using different numerical means: $\mathfrak{B}_{D}$ is calculated through a finite element interpolation while $\mathfrak{B}_{\Delta}$ presents an analytical relation Eq. (15) that is forward to implement within Matlab ${ }^{\circledR}$ for example.

\subsubsection{Modal basis}

The modal vectors are calculated using a Finite Element model made of 10000 quadratic 9-node elements. This leads to an obvious continuity 
problem since $Q_{i} \in \mathfrak{B}_{D}$ is $\mathcal{C}^{2}$-continuous over each element $\Omega \subset \mathbb{R}^{2}$ but only $\mathcal{C}^{1}$ over $\partial \Omega$, which imposes $\Delta Q_{i}$ to be a piecewise linear function $\mathcal{C}^{0}$. Such a property results in an inaccurate interpolation of the $Q_{i}$. Therefore, a concatenation of 4 quadratic elements is used to settle higher order interpolation functions that allows a $\mathcal{C}^{2}$-continuous definition of the Laplace operator. In practice, the element $\tilde{\Omega}$ is defined as $\tilde{\Omega}=\bigcup_{i=1}^{4} \Omega_{i}$. Hence, $\tilde{\Omega}$ is a 25 -nodes square element provided with $4^{\text {th }}$ order interpolation functions $\left(\mathcal{C}^{4}\right.$ continuous). It results in $\Delta Q_{i}$ to be twice continuous over $U=[0 ; a] \times[0 ; b]$ $[18]$.

\subsubsection{Spectral basis}

The $\mathcal{C}^{\infty}$-continuity of the triginometric functions along with the variable separation performed within the resolution of problem Eq. (8) leads to a straight forward analytical expression of the second derivative of $Q_{n, m} \in \mathfrak{B}_{\Delta}$ as:

$$
\Delta Q_{n, m}(x, y)=C_{n, m}\left[\cos \left(\frac{n \pi x}{a}\right) \cos \left(\frac{m \pi y}{b}\right)\right] .
$$

where $C_{n, m}=-\left(\left(\frac{n \pi}{a}\right)^{2}+\left(\frac{m \pi}{b}\right)^{2}\right)$ is a multiplicative constant.

\section{Validation from a numerical example}

\subsection{Example description}

The comparison of the two presented projection bases is performed by a numerical example. A square plate exhibiting two distinct heat sources is modeled in Abaqus ${ }^{\circledR}$ software. The finite element diffusion problem is solved numerically over a time period of $t=200 \mathrm{~s}$. The resulting temperature fields are output to a Matlab ${ }^{\circledR}$ environement and interpolated over the $300 \times 300$ monotonic grid. A random white noise is added (standard deviation is denoted $\sigma$ ) and the projector Eq. (7) is used to approximate the original temperature fields. Finally the space derivation of modes Eq. (17) and a numerical time derivation (finite differences) of the obtained temperature fields allows the use of the heat diffusion equation Eq. (1) and an approximation of the imposed heat sources.

The chosen geometry is a 3D plane-parallel plate with dimensions $(a ; a ; e)$. As shown in Fig.2, two zones are chosen to be the active heat sources of the model. The first one is rectangular and host a pyramid-shaped distribution of heat (region no.1). The second one is circular and exhibits a peak-shaped 


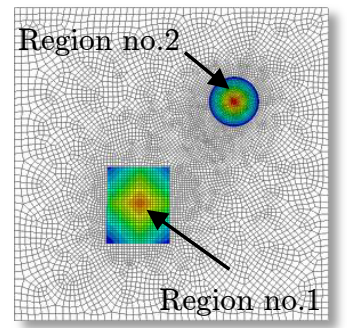

Step \#1

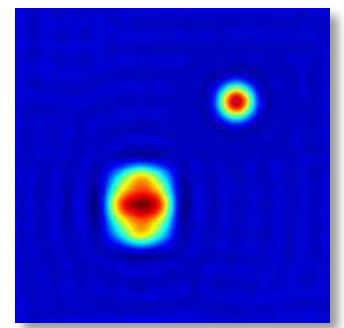

Step \#6

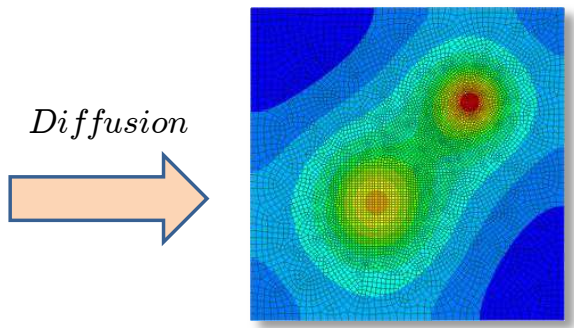

Step \#2

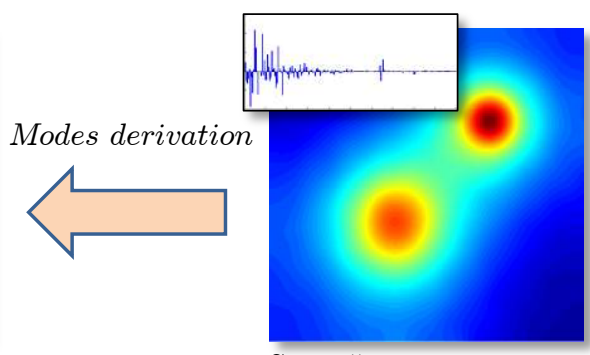

Step \#5
Interpolation

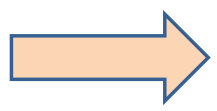

Projection

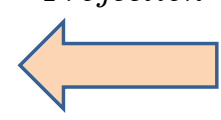

Step \#4

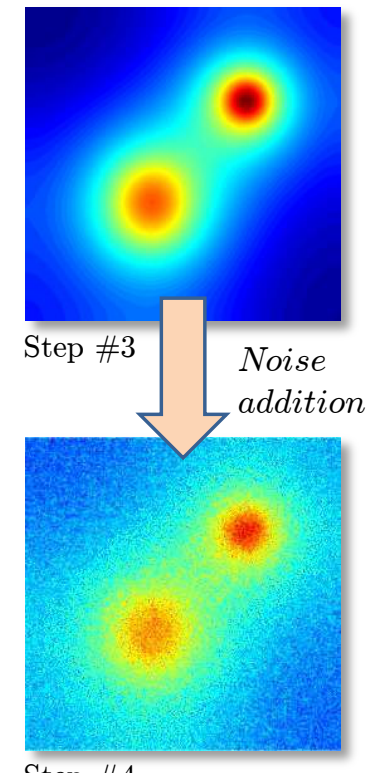

Figure 2: Solving flowchart highlighting the different steps of the proposed validation example.

distribution of heat (region no.2). The boundary conditions over the six sides of the model imposes a zero output heat flux such as:

$$
\begin{cases}\frac{\partial \theta_{k}}{\partial x}(0 ; y ; z)=\frac{\partial \theta_{k}}{\partial x}(a ; y ; z)=0 & \text { over } \partial U_{x} \\ \frac{\partial \theta_{k}}{\partial y}(x ; 0 ; z)=\frac{\partial \theta_{k}}{\partial y}(x ; a ; z)=0 & \text { over } \partial U_{y} \\ \frac{\partial \theta_{k}}{\partial z}(x ; y ; 0)=\frac{\partial \theta_{k}}{\partial z}(x ; y ; c)=0 & \text { over } \partial U_{z}\end{cases}
$$

where $\partial U_{x} \cup \partial U_{y} \cup \partial U_{z}=\partial U$. The actual dimensions are $a=100 \mathrm{~mm}$ and the thickness is $e=5 \mathrm{~mm}$. As shown in Fig.5, the magnitudes of the heat sources in region no. 1 and no.2 evolve independently with time. Finally, the computed temperature fields are output every $10 s(1 \leq k \leq 20)$ on the plate surface $(z=0)$.

The use of a $300 \times 300$ monotonic grid (denoted $\left\{x_{i} ; y_{j}\right\}$ with $\delta=x_{i}-$ $\left.x_{i-1}=y_{i}-y_{i-1}, \forall(i, j) \in[2 ; 300] \times[2 ; 300]\right)$ relies on the difference between the mesh used for mode calculation: $\mathcal{M}=\left\{u_{i} ; v_{j}\right\}$ and the one used in solving 
the thermal FE problem: $\left\{X_{i} ; Y_{j}\right\}$. As shown in Fig.3, this latter is locally refined and non-monotonic, hence the interpolation of the temperature fields over $\left\{x_{i} ; y_{j}\right\}$ assume the role of the IR camera spatial discretization (with a similar resolution).

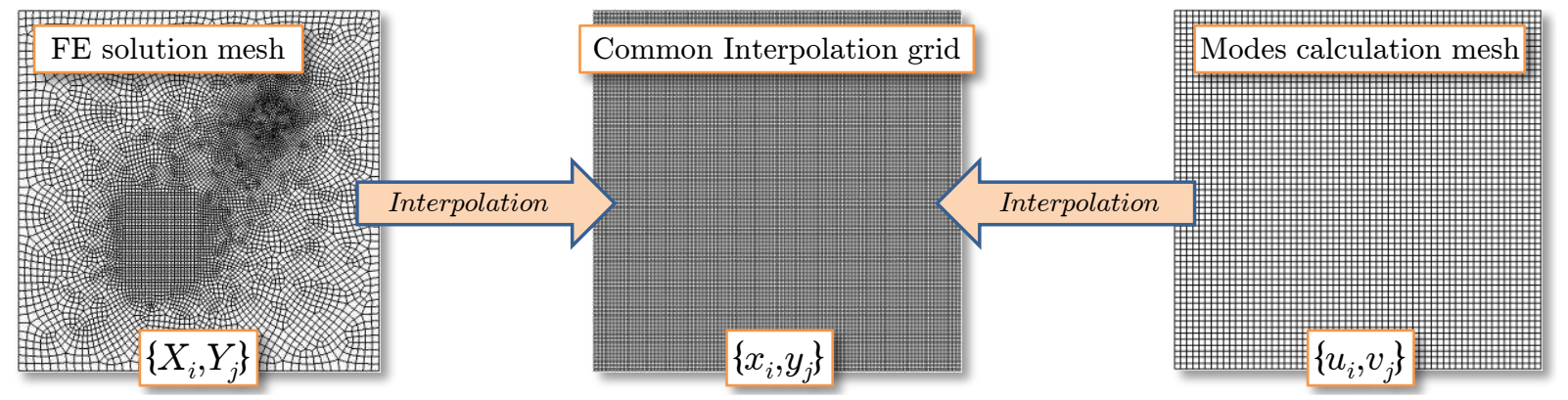

Figure 3: Meshes used for modes calculation and interpolation.

The conductivity is arbitraly set to $k=25 W \cdot m^{-1} \cdot K^{-1}$, the thermal capacity to $C=520 \mathrm{~J} . \mathrm{kg}^{-1} \cdot \mathrm{K}^{-1}$ and the mass density is $\rho=7800 \mathrm{~kg} \cdot \mathrm{m}^{-3}$.

\subsection{Results: Heat sources comparison}

Considering a noiseless $(\sigma=0)$ measured field $\theta_{k}(x, y)$, the heat sources reconstruction is performed as presented above. The truncation order is set to $N=500$ for both modal and spectral projections. The obtained heat sources are given in Fig.4. The root of mean square error (denoted $R M S$ ) is computed as a comparison between the imposed heat sources and the computed ones (denoted $w_{c h}^{\prime}$ and $\tilde{w}_{c h}^{\prime}$ respectively):

$$
R M S_{k}=\sum_{i=1}^{300} \sum_{j=1}^{300} \sqrt{\left(\tilde{w}_{c h, k}^{\prime}\left(x_{i}, y_{j}\right)-w_{c h, k}^{\prime}\left(x_{i}, y_{j}\right)\right)^{2}}
$$

It can be seen that both projections provide similar heat source reconstructions. However, the $R M S$ errors show that the projection within $\mathfrak{B}_{D}$ provide slightly closer heat sources. The time history at point 1 and 2 is plotted in Fig.5. Imposed heat sources are plotted versus the reconstructed ones (using both $\mathfrak{B}_{D}$ and $\mathfrak{B}_{\Delta}$ projection). It is noticable that the computed heat sources do not significantly vary when one projection is used or the other. Indeed, one should conclude from the presented results that, in absence of noise, modal and spectral projections provide quasi-identical heat sources at every locations and step times. 


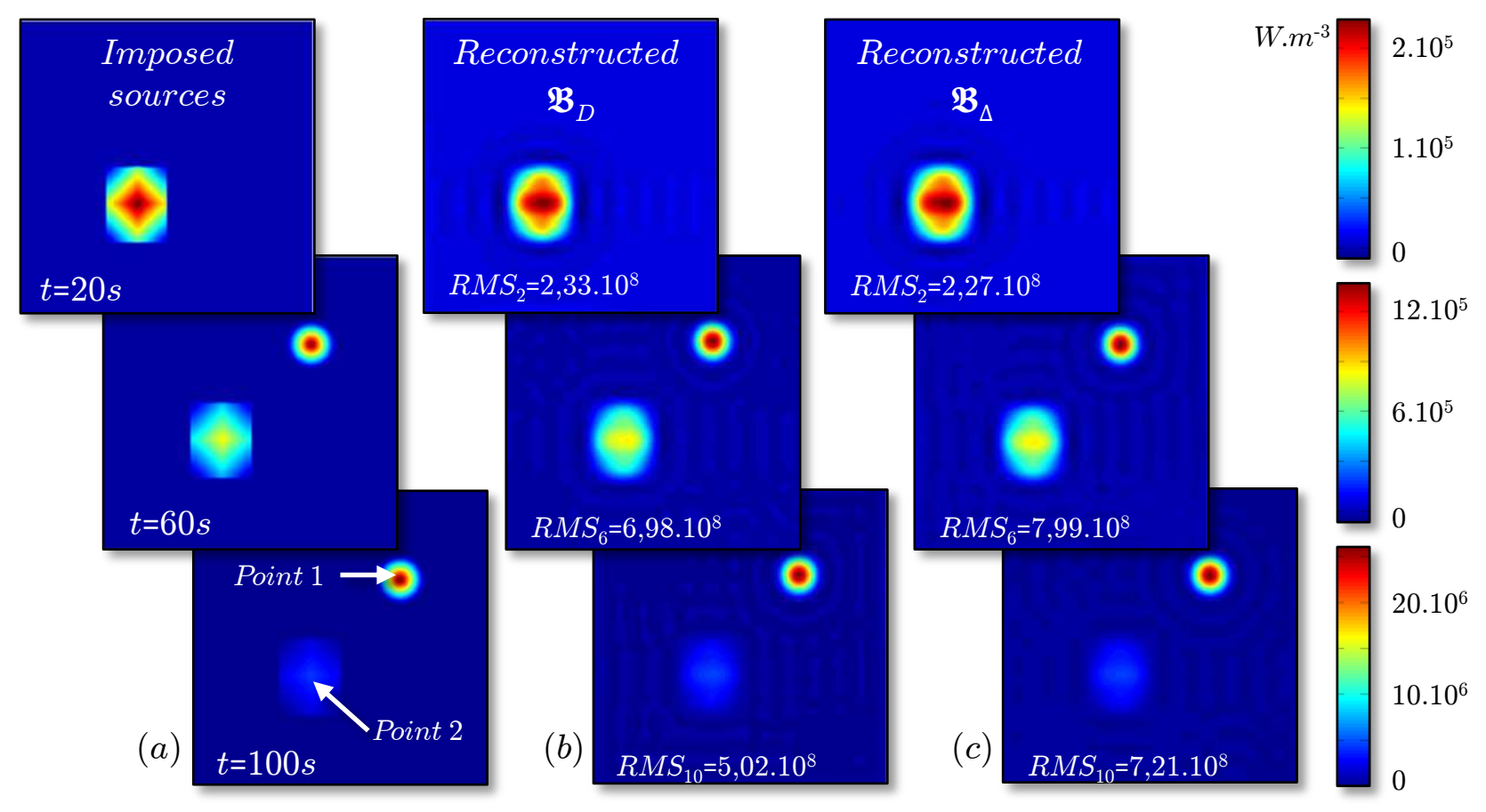

Figure 4: Imposed (a) and computed (b-c) heat sources at various step time and related RMS errors (in $\left.W \cdot m^{-3}\right)$.

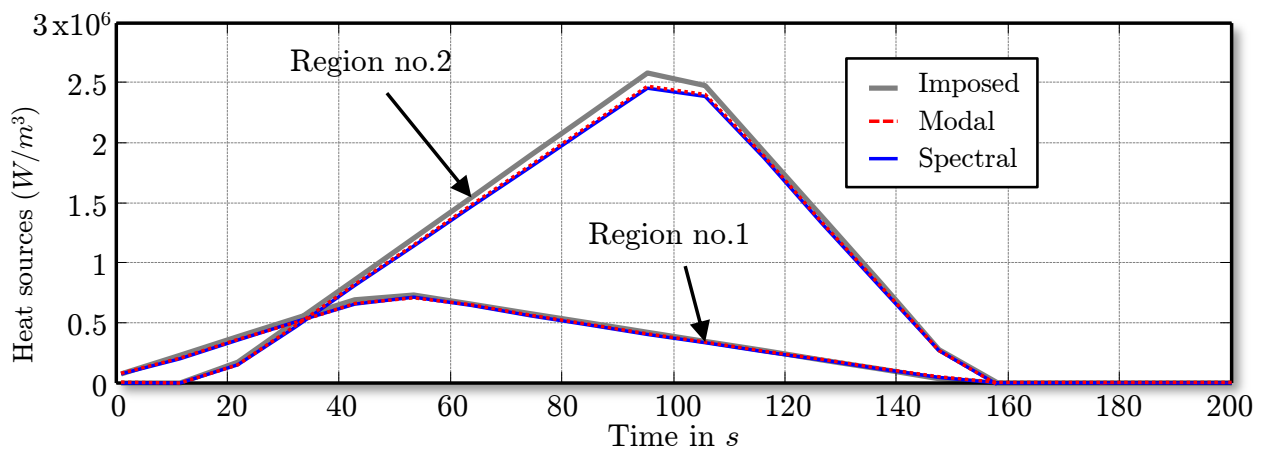

Figure 5: Time evalution of the imposed and reconstructed heat sources for both projection bases. 


\section{Discussion}

\subsection{Sensitivity to measurement noise}

The measurement noise generated by IR-camera capture may lead to significantly small signal-to-noise ratio if the recorded phenomenon involves small temperature rises. Indeed, the difficulties encountered in inverse heat transfer analysis mainly rely on the measurement noise which leads to a illposed problem which becomes very unstable to the double space derivation operator. This specific point has been adressed in many published studies $[21,3,22]$, hence the stability of the presented approach to noise is therefore highly relevant.
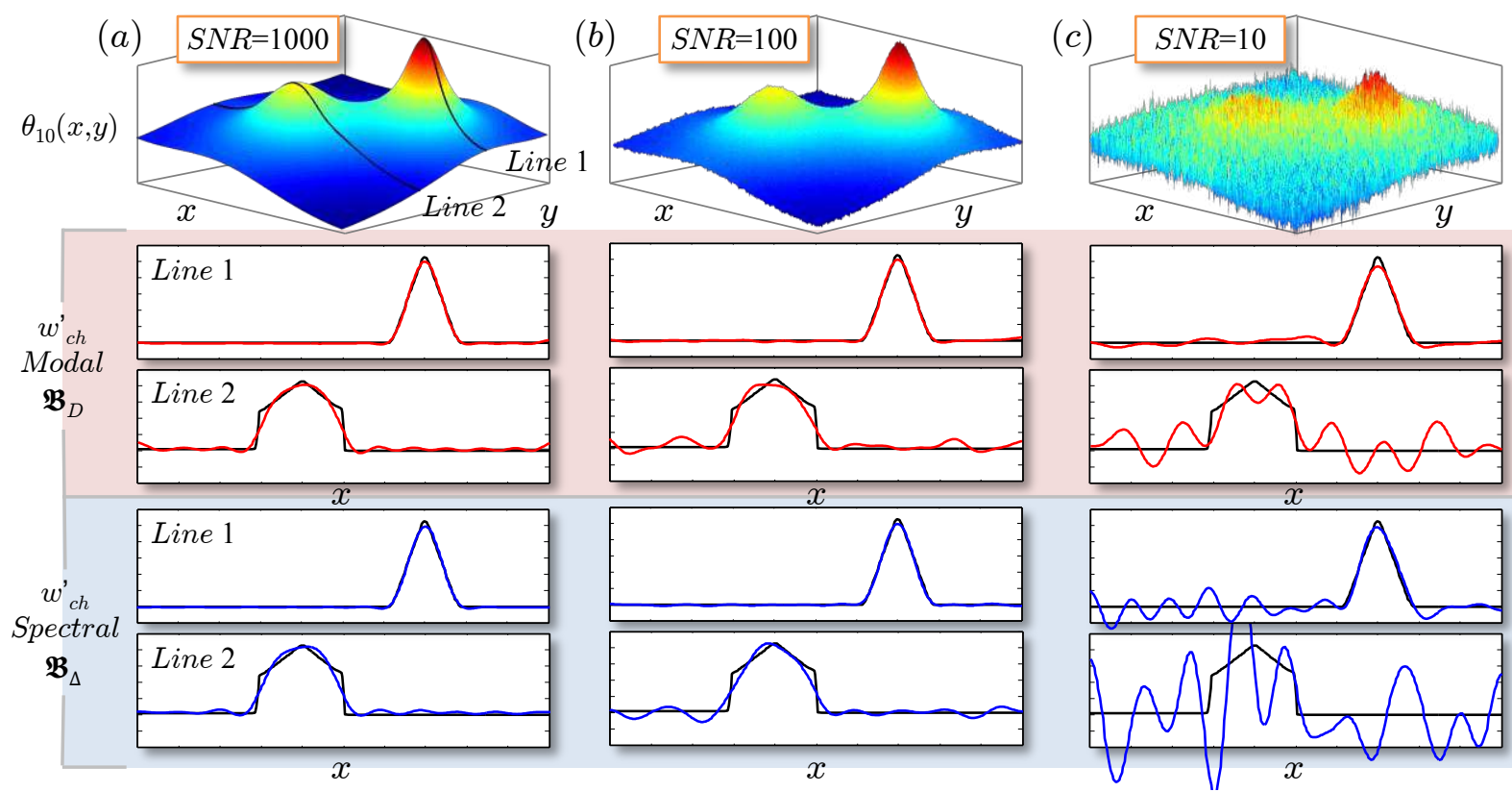

Figure 6: Heat sources reconstruction using modal and spectral projection (red lines) for increasing noises compared with the imposed sources (black lines). Graphs depicte the evolution of the reconstructed heat sources along two lines that cross the main heat sources (region no.1 and no.2 presented in Fig.2).

It has been previously shown that when the noise tends toward zero, both the projection bases provide a good approximation of the heat sources. On the other hand, the increasing of the noise magnitude leads to impair the calculations. It is therefore assumed that reconstruction error exhibits a strong 
dependence on the signal-to-noise ratio (denoted $S N R$ ). It is classically defined as:

$$
S N R=\frac{\max \left|\theta_{k}\left(x_{i}, y_{j}\right)\right|_{i j k}}{\sigma}
$$

Accordingly, a random white noise was artificially added to the numerical temperature fields with 30 increasing values of the $S N R$. Fig. 6 shows the reconstructed heat sources profiles along the two lines that cross regions no.1 and no.2. Moreover, the $R M S$ errors are computed and plotted in Fig.7.

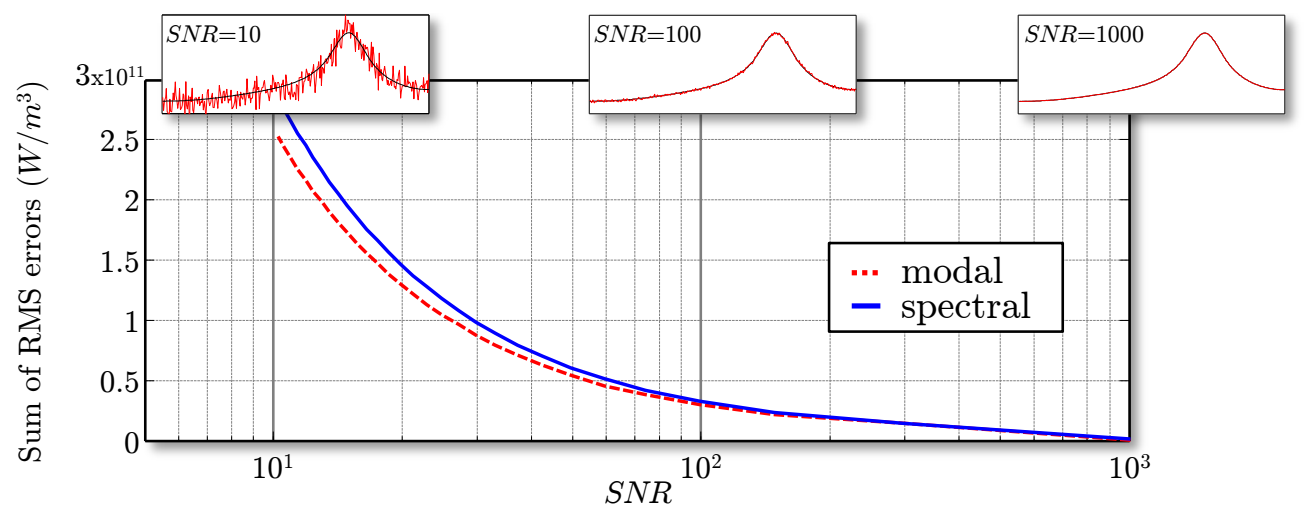

Figure 7: Comparison of the reconstruction error for increasing values of the signal-tonoise ratio.

Two conclusions can be drawn from Fig.6 and Fig.7. Firstly, as expected, the reconstruction error increases rapidly when the $S N R$ decreases. Secondly, the modal projection presents an improvement from the spectral approach since the RMS error is smaller for every noise magnitude. Though, the gap between these two reconstructions becomes particularly visible for higly noised measurements (see Fig.7 left hand side), the relative improvement is actually about $10 \%$ for all the studied $S N R$. This specific point constitues a major improvement of the proposed projection method since it allows a better heat source assessment when temperatures rises are small: e.g. small strain rates, high conductivity, early localization, vicinity of peaks...

Another relevant point can be seen in Fig.6-(c). In this case, the heat sources in region no.1 (pyramid-shaped) is actually very poorly estimated while the heat sources in region no.2 is far better approximated (especially with $\left.\mathfrak{B}_{D}\right)$. This emphasizes the shape influence on the reconstruction capability. It is well known that square-like shape leads to the use of high frequency modes and therefore impairs the Laplacian assessment. However, 
as proposed in $[17,23]$, basis enrichement can be helpfull in such case (see section 5).

\subsection{Sensitivity to truncation order}

The results presented above are obtained for a truncation order of $N=$ 500. However, this choise is arbitrary, and other values lead to different approximations of the imposed heat sources. In absence of noise, it can be shown that increasing $N$ always provides a closer approximation of the heat sources. Such an asymptotic problem imposes $R M S$ to tend toward 0 when $N$ tends toward the number of measured points (see section 2.1). One must notice that the use of interpolation (see Fig.3) prevents in practice from an exact solution of the problem. However, under the assumption of $\sigma=$ $0, R M S(N)$ is a continuously decreasing function. The ill-posed problem, settled by the addition of noise, no longer verifies this property. Therefore, the existence of an optimal truncation order (denoted $N^{*}$ ) can be assumed.

Such observations make it necessary to study, not only the influence of $N$ on the reconstruction quality, but also the influence of the signal-to-noise ratio on $N^{*}$. In practice, 40 values of $N$ were selected beween 25 (assumed here to be the smallest reasonable number of modes for a proper reconstruction) and 500 (assumed to be sufficent to obtain a very good approximation of the measured temperature field). Beside, the influence of 10 increasing values of noise on $N^{*}$ are also studied.

It can be seen from Fig.8-(a) that the modal projection provides better heat source approximations regardless of the measurement noise. As expected, in absence of noise, $R M S(N)$ continuously decrease and the modal projection slightly prevails in such a case. One may also notice that increasing the noise magnitude leads to a bigger gap between the spectral and modal projection approaches. This tends to confirm the previous observation (section 4.1): the proposed approach improves the stability to measurement noise. Fig.8-(b), shows that the optimum truncation order $N^{*}$ is always higher for the modal projection. Indeed the lower frequencies of the dynamic modes allows the use of higher truncature order even though the signal-tonoise ratio is poor, and thus provide a better temperature and heat source approximation.

\subsection{Sensitivity to convective exchanges}

The numerical example such as defined above does not include any dependency to convective exchanges over the main surface. Experimental ob- 

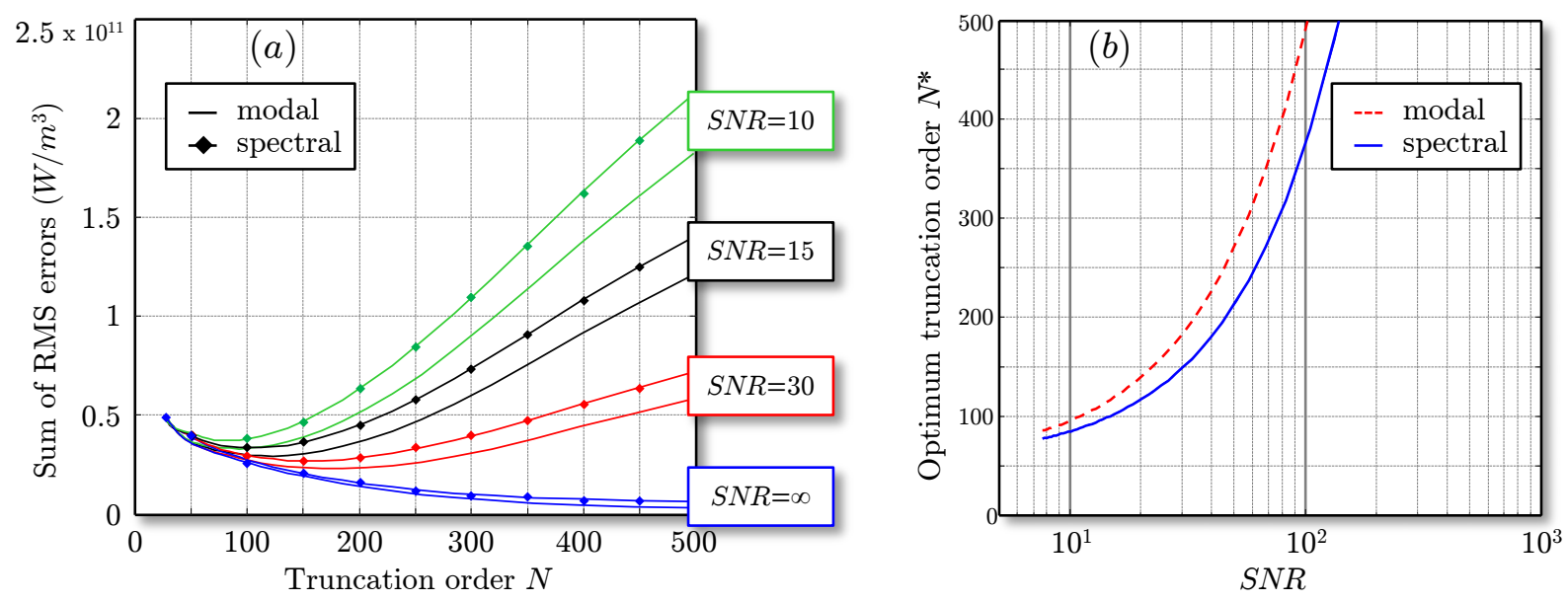

Figure 8: (a) Reconstruction error evolution versus the number of modes used for projection $N$. Four levels of noise are plotted. (b) Evolution of the optimum number of modes used in the projection versus signal-to-noise ratio.

servations classicaly lead to consider this assumption as strong and therefore requires to evaluate the sensitivity of the presented results to convective boundary conditions. Indeed, real measurement situation often lead to temperature field smoothened by convective losses over the domain borders. In such case the heat diffusion equation Eq. (22) becomes:

$$
\rho C\left(\frac{\partial \theta}{\partial t}+\frac{\theta}{\tau}\right)-k\left(\frac{\partial^{2} \theta}{\partial x^{2}}+\frac{\partial^{2} \theta}{\partial y^{2}}\right)=w_{c h}^{\prime}
$$

where $\tau$ is the time constant of the convective exchanges related to the convection coefficient $h_{z}$ in the $z$ direction as following [2]:

$$
h_{z}=\frac{\rho C e}{2 \tau}
$$

where $e$ is the thickness of the plate. The value of $h_{z}$ was set to twelve increasing values inbetween 0 and $1000 \mathrm{~W} \cdot \mathrm{m}^{-2} \cdot \mathrm{K}^{-1}$ and the RMS error between the left hand side of Eq. (22) (computed) and the right hand side of it (imposed) was monitored. The inertial and convective terms of Eq. (22) are evaluated from $\theta$ and its time derivative for both spectral and modal reconstruction. The laplacian term is assessed by the mean of either spectral or modal projection. In addition, one should notice that adding convective exchanges over the main surface does not affect the definition of the spectral 
basis such as presented in Eq. (15). Indeed the $\theta / \tau$ term clearly becomes a constant when accounting for the variable separation performed between Eq. (8) and Eq. (9) (dividing by $\theta$ ). Hence, such a formulation of the problem affects the value of the $\sigma_{n, m}$ constant but leave the modes shapes unchanged.

Fig.9-(b) shows the quality of the reconstruction from modal and spectral projection for both noisy $(S N R=50)$ and noiseless measurements.

$(a)$
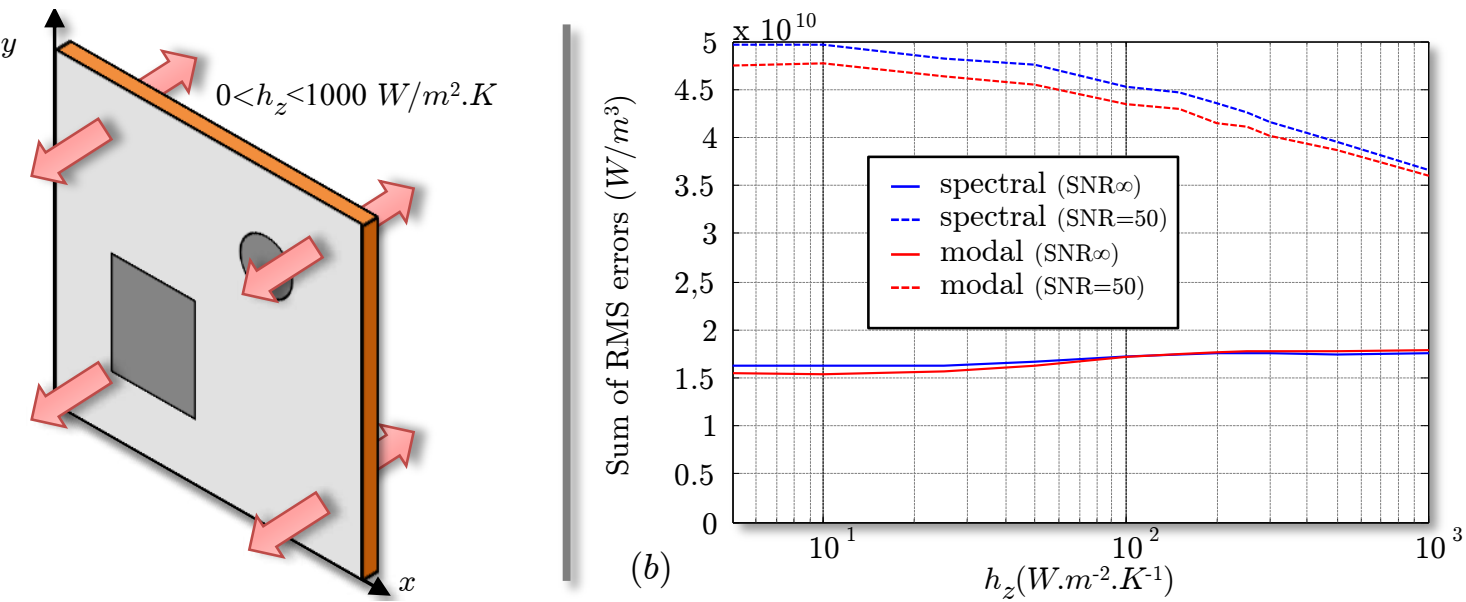

Figure 9: (a) Sketch of the FE model showing convective exchanges over the main surface. (b) Evolution of the RMS error versus $h_{z}$ for noiseless and noisy measurements.

It can be seen that for all investigated cases, the modal projection results in a lower RMS error. However the gap between the quality of the reconstructions appears to be bigger when noise is added which is consistent with the observation presented in section 4.1. It is also visible that the RMS error decreases when the convection magnitude increases. Indeed, a higher convection leads to smoothen the temperature fields and therefore decrease the influence of the Laplacian term of Eq. (22). Hence, it seems consistent to obtain a better fit with smothen fields than sharp fields.

\subsection{Boundary conditions and side effects}

Another common application of the thermal measurement is the estimation of the heat flux governed by convective exchange phenomenon. The Fourier law is classically used to model such phenomenon [2]. It offers an easy way of estimating the heat transfer coefficient $h$ from the following uni- 
directional solution:

$$
-\left.k A \frac{\partial \theta}{\partial x_{i}}\right|_{\partial U_{x_{i}}}=h_{x} \cdot A\left(T(t)-T_{e n v}\right),
$$

where $A$ stands for the surface area of the heat being transferred and $T_{\text {env }}$ is the environement temperature (here chosen to equal $T_{0}$ such as $T(t)-$ $\left.T_{e n v}=\theta\right)$. Hence, one may notice that assuming the space-derivability of the measured temperature field, $h_{x}$ can be assessed in a straight forward manner as:

$$
h_{x}=-\left.\frac{k}{\theta} \frac{\partial \theta}{\partial x}\right|_{x=a} .
$$

As depicted in Fig.10-(a) two convective exchanges are added to the finite element model definition and lead to thermal losses through $\partial U_{x}$ and $\partial U_{y}$. The imposed magnitudes are $h_{x}=100 \mathrm{~W} \cdot \mathrm{m}^{-2} \cdot \mathrm{K}^{-1}$ and $h_{y}=50 \mathrm{~W} \cdot \mathrm{m}^{-2} \cdot \mathrm{K}^{-1}$ over two cross section areas of $100 \times 5 \mathrm{~mm}^{2}$. All other parameters remain unchanged.
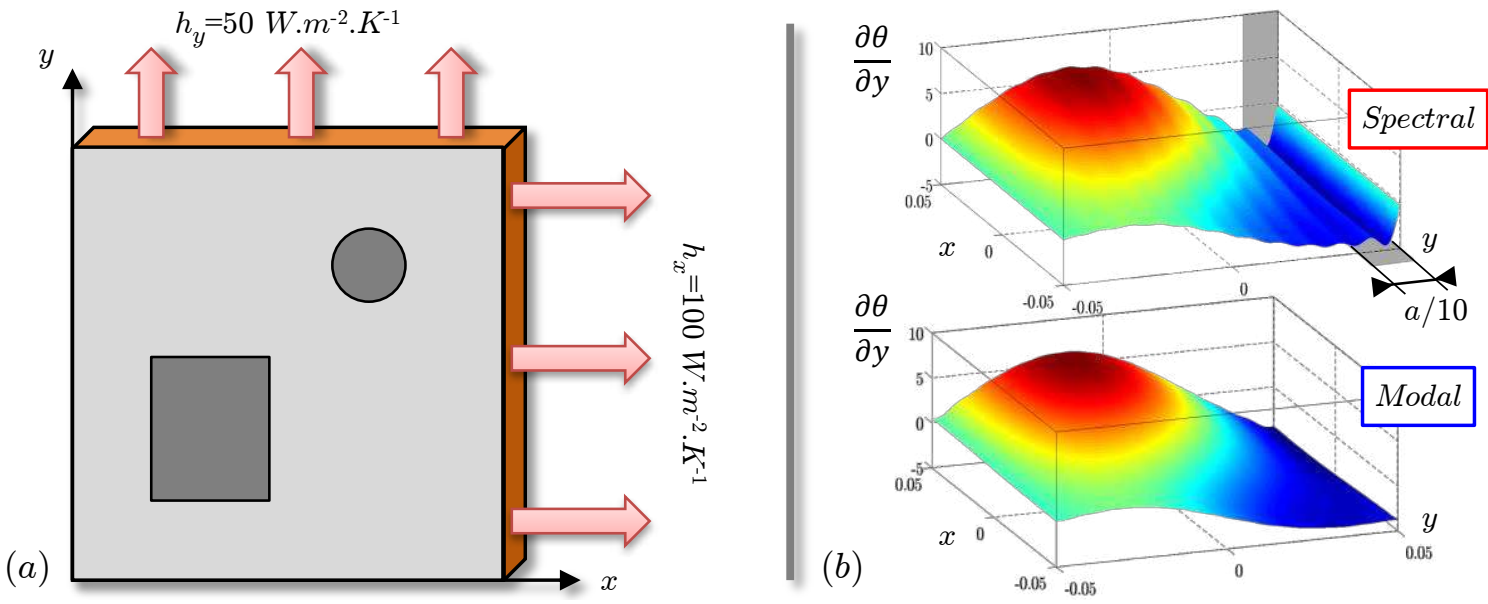

Figure 10: (a) Sketch of the FE model showing both heat sources and the heat tranfers over two sides of the domain $U$. (b) Shape of the $x$-derivative of the projected temperature fields in $\mathfrak{B}_{\Delta}$ and $\mathfrak{B}_{D}$ emphasizing side effects at $t=200 \mathrm{~s}$.

As pointed by $[2,15]$, one of the main disadvantage of the spectral decomposition is the bad quality of the approximation at the borders. Local divergences of the RMS error arises (see Fig.10-(b)) due to the definition of the spectral basis used in Eq. (8). Indeed, $\mathfrak{B}_{\Delta}$ is built under the assump- 
tion of Neumann's conditions and is thus not suited to model convective exchanges. In addition, it must be emphasized that, without a priori knowledge of the heat transfer coefficients $h_{x}$ and $h_{y}$, it is pointless to re-settle the Laplace problem, Eq. (8) in order to build a spectral basis that accounts for either Dirichlet's or mixed boundary conditions. Indeed, the fact that $\mathfrak{B}_{\Delta}$ is close-to-physics become a major problem when this physics is only partially known.

It can be seen in Fig.11 that using the spectral projection disables any straight identification of the heat transfer coefficients. However, the use of a linear approximation over the vicinity of the borderline allows an approximation of $h_{x}$ and $h_{y}$. Fig.11 shows the results obtained for a linear interpolation from $x_{i}=a / 10$ to $x_{i}=a$ (see Fig.10-(b)).

On the other hand, the use of modal projection provides good approximations of the imposed heat transfer coefficients and apprears, once again, to be less noise-sensitive (Fig.11). The modal basis is build from dynamics considerations and is therefore not as close-to-physic as the sprectral one. However, the imposed boundary conditions (free translation along $z$ and free rotation around $x$ and $y$ ) results in the absence of significant side effects (Fig.10-(b)).

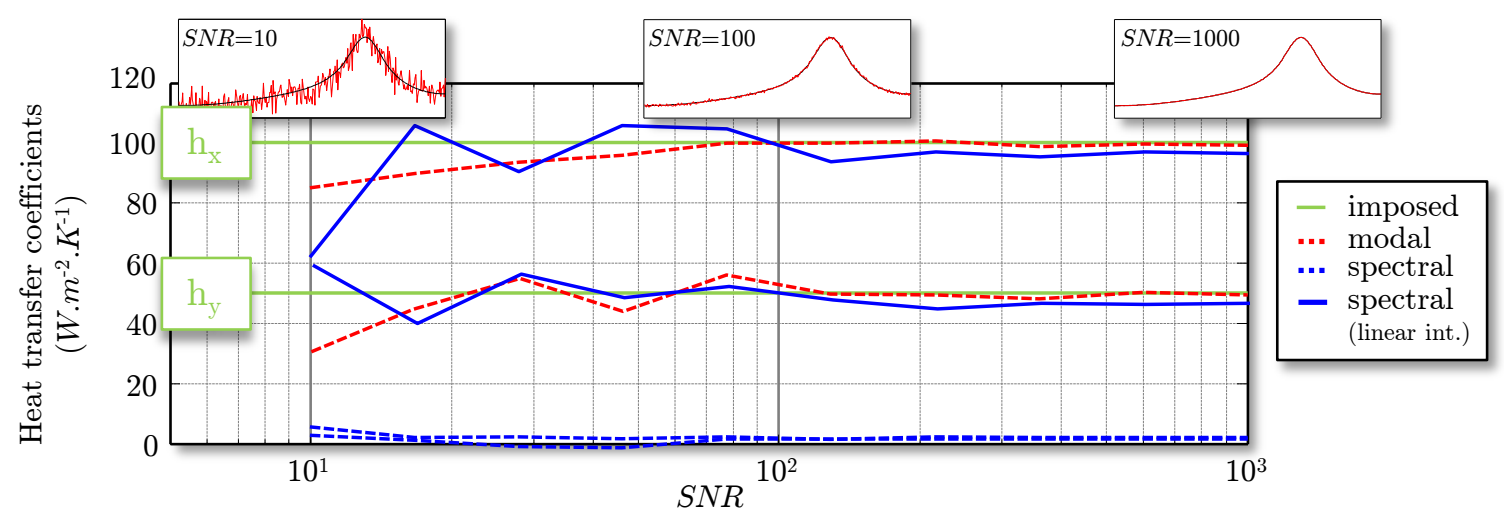

Figure 11: Evolution of the identified heat transfer coefficents $\left(h_{x}\right.$ and $\left.h_{y}\right)$ versus signalto-noise ratio.

\section{Basis enrichment}

As noticed above, the heat source reconstruction procedure is very shapesensitive. Indeed, Fig.6-(c) shows that the reconstruction improves as the 
shape of the heat source gets closer to the mode shapes (e.g. curves rather than sharp angles). The basis enrichement procedure presented in $[13,23]$ proposes to insert tailor-made modes into the natural basis in order to describe specifically the boundary conditions (and thus solve the aforementioned side effects problem). Yet, trivial mathematical considerations in finite dimension show that any orthogonal basis $\mathfrak{B}$ which is added modes $\tilde{Q}_{i}$ looses its orthogonality property but still defines a unique projection of $\theta_{k}(x, y)$ within $\tilde{\mathfrak{B}}=\mathfrak{B} \bigcup\left\{\tilde{Q}_{i}\right\}$. Indeed, it is shown in [21] that Eq. (7) is the solution that minimize the quadratic gap between the measured field and the projected approximation.

The hereby proposed procedure relies on the use of tailor-made modes (called thermomechanical modes) to describe the heat sources shapes themselves. A similar procedure has already been used in shape metrology with significant improvements [17]. The main difficulty is that users may only have an approximated knowledge of the heat sources shapes (for example from strain localization, geometrical specificities, etc) but the measured and projected data are temperature fields. Moreover, though the shapes of the heat sources can be roughly known a priori, their magnitudes requires the assumption of constitutive equations. Hence, the construction of thermomechanical mode requires (i) the use of a heat diffusion solver and (ii) a normalized heat mode. One can then use a Gram-Schmidt algorithm to orthogonalize the so-obtained base if needed but this is not mandatory since a projection within the dual basis (as proposed in Eq. (7)) is still available.

In the presented numerical example, the addition of tailor-made modes that describes the heat sources in region no.1 and no.2 is performed from the actual knowledge of their shapes. Two enhanced bases are assembled using thermomechanical modes build from $i$ ) the exact heat sources shapes $\left(\tilde{Q}_{1}\right.$ and $\left.\tilde{Q}_{2}\right)$ and from $\left.i i\right)$ an approximation of it $\left(\tilde{Q}_{1}^{a}\right.$ and $\left.\tilde{Q}_{2}^{a}\right)$. Hence, the improvements achieved from using these enriched bases can be verified and quantified. In this section, only the results from enhanced modal projections are presented.

As shown in Fig.12-(ab), four modes are built from the shapes of the heat sources no.1 and no.2 (two from the exact shape and two from the approximated shapes). Unitary heat sources are input and their temperature diffusion after $10 \mathrm{~s}$ are monitored (Fig.12-(cd)). These modes are slipped 

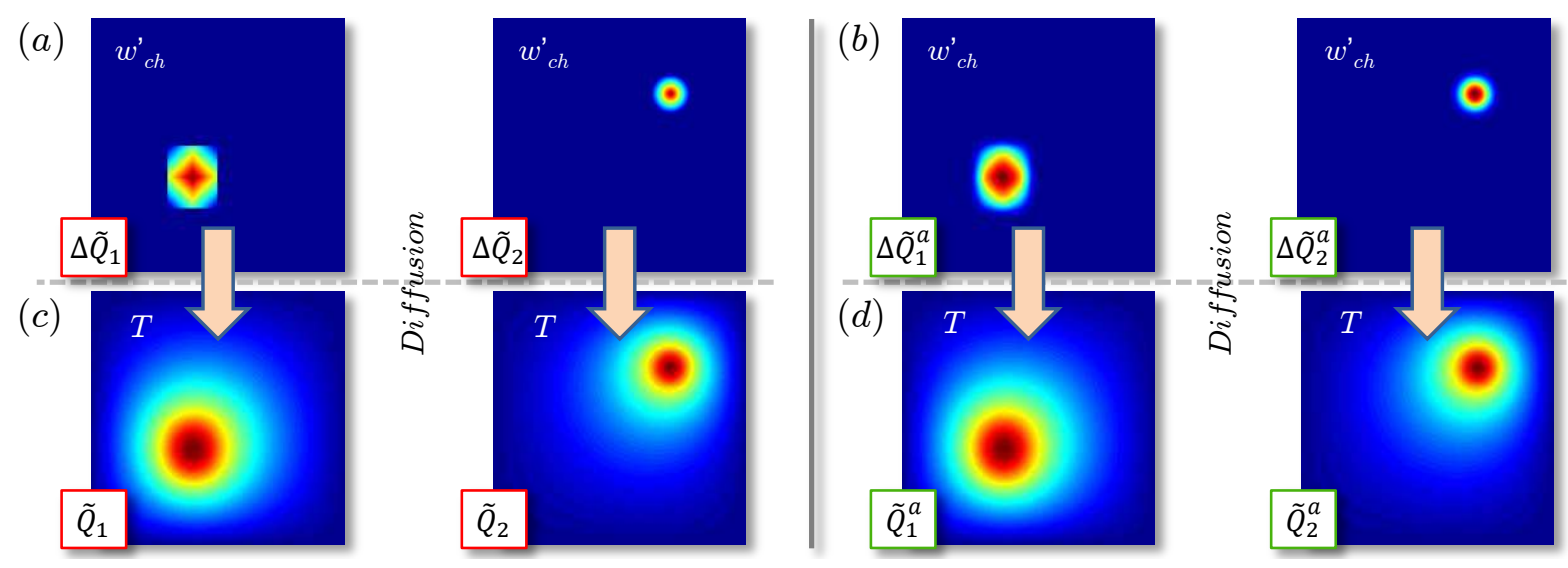

Figure 12: (a) Exact source shapes. (b) Approximated source shapes. (c) Modes $\tilde{Q}_{1}$ and $\tilde{Q}_{2}$ corresponding to the temperature fields after $10 \mathrm{~s}$ diffusion. (d) Modes $\tilde{Q}_{1}^{a}$ and $\tilde{Q}_{2}^{a}$ corresponding to the temperature fields after $10 \mathrm{~s}$ diffusion of the approximated heat sources.

after the first rigid mode $\left(Q_{1}=1 \forall(x, y) \in \mathbb{R}^{2}\right)$ so that:

$$
\begin{aligned}
\tilde{\mathfrak{B}}_{D} & =\left(Q_{1}, \tilde{Q}_{1}, \tilde{Q}_{2}, Q_{2}, \ldots, Q_{N}\right) \\
\tilde{\mathfrak{B}}_{D}^{a} & =\left(Q_{1}, \tilde{Q}_{1}^{a}, \tilde{Q}_{2}^{a}, Q_{2}, \ldots, Q_{N}\right) .
\end{aligned}
$$

The so-obtained bases are reset orthogonaly using a Gram-Schmidt algorithm. Once again the temperature modes are normalized using an infinite norm. The approximated set of thermomechanical modes is built from eroded shapes of the heat sources. A Gaussian filtering-window is used over the exact heat source shapes (Fig.12-(b)). This accounts for the fact that a priori knowledge of the heat source shapes may only be approximated.

Fig. 13 shows the sum of reconstruction error versus signal-to-noise ratio. It can be seen that basis enrichment does improve the quality of the heat source calculation but only if the $S N R$ is over 25 for $\tilde{\mathfrak{B}}_{D}$ and 32 for $\tilde{\mathfrak{B}}_{D}^{a}$. Indeed, the natural basis remains the most able to describe the shape when measurement noise is massive. Another interesting feature depicted in Fig.13 is the prevailing of exact modes $\tilde{Q}_{i}$ over the approximated ones $\tilde{Q}_{i}^{a}$. An exact knowledge of the heat sources shapes leads to a closer assessment of their magnitudes. However, the modes built from an approximated knowledge of the shapes allows a better reconstruction than using the non-modified natural basis, in the case of small noise magnitudes. It can therefore be pointed out 


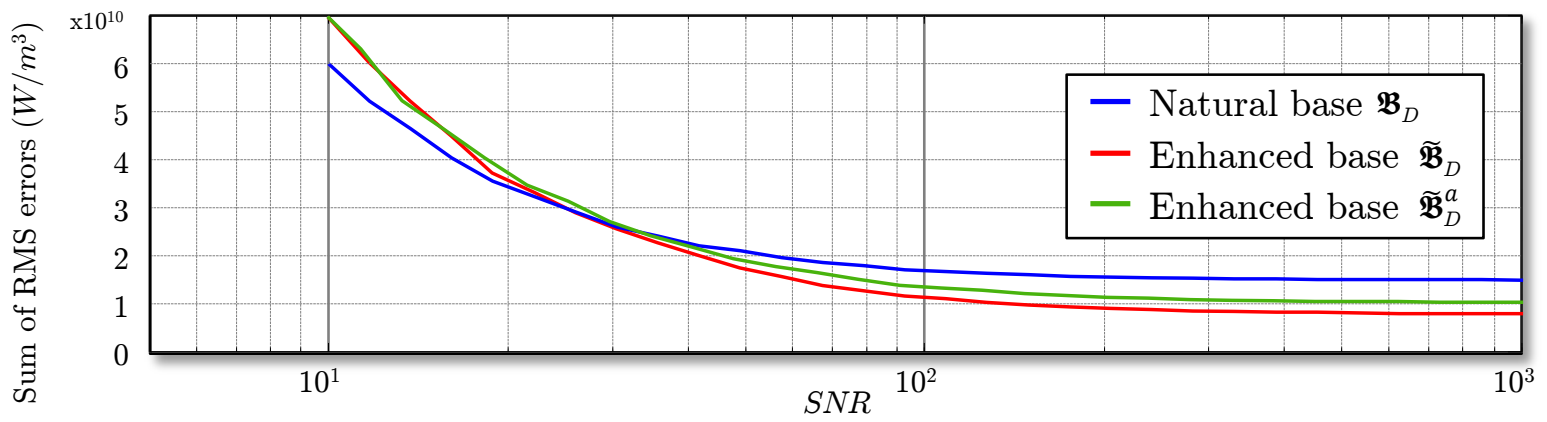

Figure 13: Sum of reconstruction error versus signal-to-noise ratio for $\mathfrak{B}_{D}, \tilde{\mathfrak{B}}_{D}$ and $\tilde{\mathfrak{B}}_{D}^{a}$.

that, if the $S N R$ is reasonnably high enough, any a priori knowledge of the heat sources shapes (approximated shapes and unitary magnitude) improves the heat source reconstruction.

This can be seen and interpreted in Fig.14. The modal spectrum are depicted from projections at $t=100 s$ using $\mathfrak{B}_{D}, \tilde{\mathfrak{B}}_{D}$ and $\tilde{\mathfrak{B}}_{D}^{a}$. It can be seen that using an enriched basis leads to gather the high magnitude modes at very low frequencies (i.e. the left hand side of the spectrum). Accordingly the double space-derivation required by the Laplace term evaluation is not impaired by high frequencies modes and the numerical resolution provides a better match.
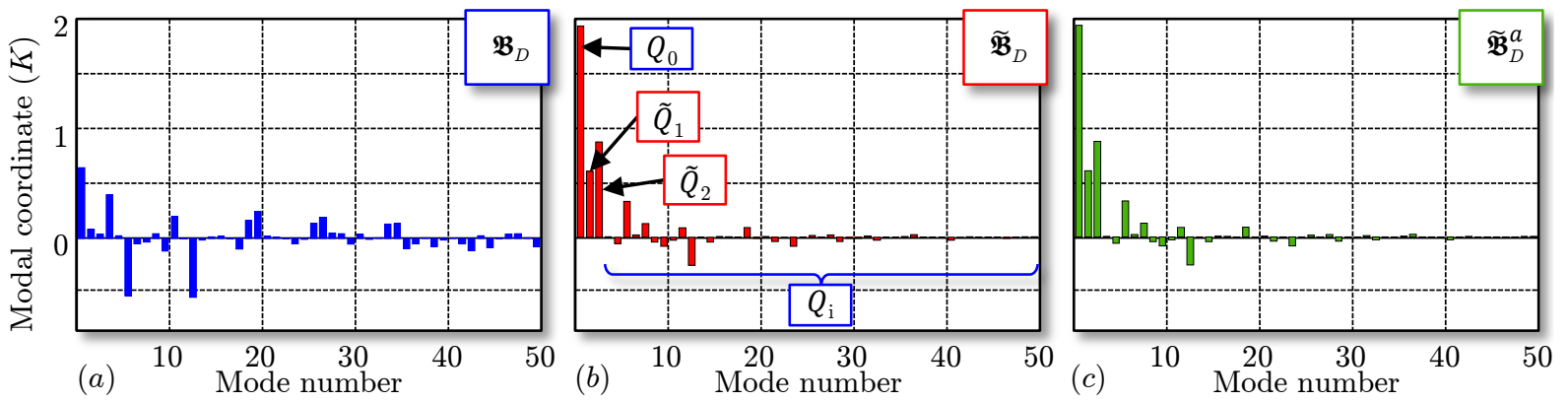

Figure 14: Projection spectrums at $t=100 \mathrm{~s}$ using natural basis and the two enhanced basis $\left(\tilde{\mathfrak{B}}_{D}\right.$ and $\left.\tilde{\mathfrak{B}}_{D}^{a}\right)$.

As a matter of fact, the diffusion nature of the heat equation (1) allows to build suitable temperature modes from rough estimate of the heat sources shapes (see Fig.12-(cd)). In addition, one may think of plenty of formulations to enhance such an inverse thermo-mechanical problem espe- 
cially a combined approach using modes built from a priori knowledge of the heat sources shapes and/or boundary conditions, and/or noise distribution, and/or microstructural consideration, etc. Indeed, the use of coupled thermal-kinematic measurement offers a wide range of information on material deformation processes that could be used to enhance the projection procedure.

\section{Conclusion}

The presented study shows that regardless of how far from physics the modal projection is, it allows significant improvements in heat source estimation. It appears to be less sensitive to noise and side effects and thus allows a more accurate characterisation of calorific effects from temperature full-field measurements. In addition, the paper proposes to enhanced the projection bases by the means of thermomechanical modes possibly coming from strain measurements. This latter aspect has to be detailled more deeply in further publications. It is also noticable that building the modes from structual dynamics allows to account various geometry such as disk, cylinder, hollowed plates, notched specimen, etc since a very simple FE calculation is required.

Boundary conditions constitutes a classical problem of thermal field projection methods. It is noticable that the presented approach allows to impose a wide range of them. Indeed the mechanical formulation of the problem leads to generate the modal basis from Finite Element calculations and hence allows to impose either displacement, forces or velocity which all have thermal equivalences.

The spectral basis, is built from thermal considerations and thus is closer to physics. However, it appears to be less efficient in the presented numerical tests. The formulation of this basis tested in this paper is the classical one and not a enhanced one such as proposed by [13, 23]. Indeed, it should be accounted that several flaws of such a decomposition have already be pointed out in many former studies and improvement procedures has been proposed and implemented [15] .

Though, such a complex filtering approach is probably unnecessary when measurements are clear from noise and exhibit sufficient space resolution, it constitutes a robust alternative in ill-posed inverse thermal problems. It must also be pointed that the presented numerical implementation leads to quick calculation since the modal basis is inverted once and for all. Processing a dozen images require less than a minute with an up-to-date computed. 
Applying the presented procedure to actual experimental measurements is the main perspective of the study. Indeed, the presented investigation validates the ability of modal filtering approach to provides a robust and reliable estimation of the thermal heat sources.

[1] A. Chrysochoos, O. Maisonneuve, G. Martin, H. Caumon, J.-C. Chezeaux, Plastic and dissipated work and stored energy, Nuclear Eng. and Design 114 (1989) 323-333.

[2] A. Chrysochoos, H. Louche, An infrared image processing to analyse the calorific effects accompanying strain localisation, International Journal of Engineering Science 38 (2000) 1759-1788.

[3] H. Louche, A. Chrysochoos, Thermal and dissipative effects accompanying Lüders band propagation, Materials Science and Engineering: A A307 (2001) 15-22.

[4] H. Louche, P. Vacher, R. Arrieux, Thermal observations associated with the Portevin-Le Chatelier effect in an Al-Mg alloy, Materials Science and Engineering: A 404 (2005) 188-196.

[5] A. Chrysochoos, B. Wattrisse, J.-M. Muracciole, Y. El Kaïm, Fields of stored energy associated with localized necking of steel, Journal of Mechanics of Materials and Structures 4 (2009) 245-262.

[6] S. Dumoulin, H. Louche, O. Hopperstad, T. Børvik, Heat sources, energy storage and dissipation in high-strength steels: Experiments and modelling, European Journal of Mechanics - A/Solids 29 (3) (2010) 461474 .

[7] W. Xiaoyan, Z. Dongsheng, Q. Yu, L. Jieyan, F. Nie, Improving the peak wavelength detection accuracy of Sn-doped, H2-loaded FBG high temperature sensors by wavelet filter and Gaussian curve fitting, Sensors and Actuators / A 174 (2012) 91-95.

[8] F. Bozzoli, G. Pagliarini, S. Rainieri, Experimental validation of the filtering technique approach applied to the restoration of the heat source field, Experimental Thermal and Fluid Science 44 (2013) 858-867. 
[9] Z. Yi, D. Murio, Source terms identification for the diffusion equation, H.R.B. Orlande, editor, Proceedings of the $4^{\text {th }}$ Int. Conf. on Inverse Problems in Engineering :Theory and Practice 1 (2002) 99-106.

[10] F. Chuli, Q. Chunyu, Wavelet and error estimation of surface heat flux., J. Computational and Applied Mathematics 150 (2003) 143-155.

[11] A. Wong, G. Kirby, A hybrid numerical/experimental technique for determining the heat dissipated during low cycle fatigue, Eng. Fract. Mech. 37 (3) (1990) 493-504.

[12] J. Kaipio, E. Somersalo, Statistical inverse problems : Discretization, model reduction and inverse crimes, Journal of Computational and Applied Mathematics 198 (2) (2007) 493-504.

[13] C. Doudard, S. Calloch, F. Hild, P. Cugy, A. Galtier, A probabilistic twoscale model for high cycle fatigue life predictions, Fatigue and Fracture of Engineering Materials and Structures 28 (2005) 279-288.

[14] Y. Candau, Solving inverse heat diffusion problem with discrete wavelet transform, Inverse Problems in Science and Engineering 13 (4) (2005) 329-339.

[15] A. Neveu, K. El-Khoury, B. Flament, Simulation de la conduction non linéaire en régime variable: décomposition sur les modes de branche, Int. J. Therm. Sci (in french) 38 (1) (1999) 289-304.

[16] N. Renault, S. Andréa, D. Maillet, C. Cunat, A spectral method for the estimation of a thermomechanical heat source from infrared temperature measurements, International Journal of Thermal Sciences 49 (2010) 1394-1406.

[17] P.-A. Adragna, S. Samper, M. Pillet, H. Favrelière, Analysis of shape deviations of measured geometries with a modal basis, Journal of Machine Engineering. Manufacturing Accuracy Increasing ProblemsOptimization 6 (1) (2006) 95-102.

[18] G. Le Goic, H. Favrelière, S. Samper, F. Formosa, Multi scale modal decomposition of primary form, waviness and roughness of surfaces, Scaning 33 (5) (2011) 332-341. 
[19] W. Wang, J. Mottershead, C. Sebastian, E. Patterson, Shape features and finite element model updating from full-field strain data, International Journal of Solids and Structures 48 (2011) 1644-1657.

[20] M. Boas, Mathematical Methods in the Physical Sciences, John Wiley and Son, 1983.

[21] C. Le Niliot, P. Gallet, Infrared thermography applied to the resolution of inverse heat conduction problems: recovery of heat line sources and boundary conditions, Revue Générale de Thermique 37 (1998) 629-643.

[22] T. Boulanger, A. Chrysochoos, C. Mabru, A. Galtier, Calorimetric analysis of dissipative and thermoelastic effects associated with the fatigue behavior of steels, International Journal of Fatigue 26 (2004) 221-229.

[23] C. Doudard, S. Calloch, F. Hild, S. Roux, Identification of heat source fields from infrared thermography: Determination of 'self-heating' in a dual-phase steel by using a dog bone sample, Mechanics of Materials 42 (2010) 55-62. 